\title{
Mechanisms in Cognitive Psychology: What Are the Operations?
}

\author{
William Bechtel†
}

Cognitive psychologists, like biologists, frequently describe mechanisms when explaining phenomena. Unlike biologists, who can often trace material transformations to identify operations, psychologists face a more daunting task in identifying operations that transform information. Behavior provides little guidance as to the nature of the operations involved. While not itself revealing the operations, identification of brain areas involved in psychological mechanisms can help constrain attempts to characterize the operations. In current memory research, evidence that the same brain areas are involved in what are often taken to be different memory phenomena or in other cognitive phenomena is playing such a heuristic function.

1. Introduction. Contemporary interest in mechanism and mechanistic explanation has been fueled primarily by investigations of experimental biology (molecular and cell biology, biochemistry, neurobiology), in which there are few appeals to laws and in which the term mechanism is widely applied (Bechtel and Richardson 1993; Machamer, Darden, and Craver 2000; Darden 2005; Bechtel 2006; Craver 2007). In psychology there is likewise a paucity of laws (Teigen 2002). Robert Cummins (2000) observes both that in psychology laws are often referred to as effects and that effects typically characterize phenomena in need of explanation rather than providing explanations. The spacing effect, discovered by Hermann Ebbinghaus (1885), describes the fact that learning which is spaced results in greater recall than massed learning but offers no explanation of this finding. In psychology, as in the biological sciences, the term most frequently invoked in presenting explanations is mechanism.

My focus is on mechanistic explanations advanced in cognitive psychology and on ways in which these explanations differ from those found in the biological disciplines. In particular, I focus on the special challenges

$\dagger$ To contact the author, please write to: Department of Philosophy, 0119, University of California, San Diego, La Jolla, CA 92093-0119; e-mail: bill@mechanism.ucsd.edu. \$I thank Lindley Darden for very helpful comments and suggestions.

Philosophy of Science, 75 (December 2008) pp. 983-994. 0031-8248/2008/7505-0041 \$10.00 Copyright 2008 by the Philosophy of Science Association. All rights reserved. 
confronting cognitive psychologists in developing mechanistic explanations. Mechanistic explanation involves identifying a mechanism responsible for a given phenomenon and characterizing the working of the mechanism by decomposing it into component parts and operations and showing how they are organized and orchestrated. In most cell and molecular mechanisms, including those operative in the brain, the component operations involve material transformations (e.g., moving ions or oxidizing a substrate), whereas in most mechanisms proposed in cognitive psychology it is information that is transformed. This presents a major challenge for psychologists: how to identify the information-processing operations within the mechanism. Often the decompositions that cognitive psychologists propose analyze a phenomenon such as memory into subtypes (e.g., explicit vs. implicit), not into operations within a mechanism. In the past 20 years, as a result of new investigatory techniques, such as functional neuroimaging, cognitive psychologists have been able to identify brain areas involved in various cognitive tasks. I will conclude by showing how this helps constrain, but does not itself solve, the problem of how to characterize the operations in psychological mechanisms.

2. Material Transformations versus Processing Information. A challenge researchers confront in developing accounts of mechanisms in any field is identifying the operations through which the mechanism realizes the phenomena of interest. All cells in living organisms metabolize foodstuffs to generate adenosine triphosphate (ATP), the major energy source utilized by other mechanisms, such as those involved in muscle contraction or in synthesizing proteins. Identifying the operations in this mechanism was facilitated by identifying possible intermediate substances produced in the catabolism of sugar to carbon dioxide and water and demonstrating the occurrence of the reactions in which these intermediates figured. One of the great challenges researchers faced was determining how the energy released in various oxidation reactions was transferred to ATP, a problem that was solved by discovering that it was not maintained in a chemical intermediate but as a proton gradient across a membrane. Researchers then directed their attention to how this gradient was established and utilized in generating ATP. Likewise, in discovering the mechanism of protein synthesis, researchers were guided in part by identifying the different forms of RNA that figure in the process. Discovering the various substances that are produced or transformed in operations provides a powerful heuristic for characterizing the operations themselves (Bechtel 2006).

Mental phenomena, however, are not characterized in terms of transformations of physical material. Rather, they are commonly characterized according to their role in coordinating the behavior of an organism in 
response to sensory information, including information acquired long ago in the past. Behaviorist theories attempted to explain regularities in terms of processes of learning. These were thought to be sufficiently similar across species and tasks that it was unnecessary to posit multiple types of mental operations. The cognitive turn against behaviorism was motivated in part by the recognition that simple learning procedures alone could not account for complex patterns of behavior in language use (Chomsky 1956, 1959), purposive action (Miller, Galanter, and Pribram 1960), or even perception (Bruner and Goodman 1947) and in part by the formulation of the idea of information-processing mechanisms (Hebb 1949; Broadbent 1958; Neisser 1967). In such mechanisms, information not only can be passed down a channel (Shannon 1948) but it can also be transformed by filtering, amplifying, or replacing elements of the signal (Broadbent 1958).

Information-processing mechanisms operate on representations, and it is useful to distinguish the vehicle of representation (bits in a computer or action potentials in the brain) from the content (the condition for purposive action or the features of an object). In explaining behavior, the interest is not in the vehicles but in the informational content. The operations must enable the informational content needed to guide behavior to be available to the output systems. The intermediate processes in transforming informational contents, however, are far less easy to track than those involved in material transformations. In the latter case, researchers can try to inhibit a transformation process and detect the buildup of intermediates or insert postulated intermediates into the reaction to determine whether downstream reactions proceed as expected (Bechtel and Richardson 1993). As I will discuss later, if researchers have access to the representational vehicles, that can provide important guidance to the information-processing operations occurring in the mechanism. For most of the period since the creation of cognitive psychology around the middle of the twentieth century, however, cognitive psychologists did not have access to the representational vehicles in the brain but had to rely on behavior to figure out both the parts and operations. This meant using patterns of response, including errors, and behavioral measures, such as reaction times, to identify the operations within the mechanism. Such indirect measures ruled out candidate information-processing accounts (Sternberg 1966) but did not provide information about the nature of the operations being performed.

3. Phenomenal Decomposition versus Mechanistic Decomposition. I noted above the critical role decomposition plays in coming to understand a mechanism, since it is the organized parts and operations within a mechanism that explain what the mechanism does. Many studies in cognitive 
psychology do report decompositions, but as a brief examination of research on memory reveals, this research has tended to decompose phenomena into different types rather than decomposing a particular phenomenon into its operations.

For example, one of the first contributions of cognitive psychology was to rediscover and further develop the distinction William James (1890) made between primary and secondary memory and renaming them shortterm (later working) memory and long-term memory. George Miller (1956) established that a maximum of seven (plus or minus two) items could be retained in memory through a process of active rehearsal. L. R. Peterson and M. J. Peterson (1959) demonstrated severe decay of such stored information after delays of 3-9 seconds during which subjects were required to perform an interfering task (counting backwards by threes). Nancy Waugh and Donald Norman (1965) found greater forgetting the further back in a list the recalled item was. These limitations were not present when items were sufficiently rehearsed to enter long-term memory. George Sperling (1960) determined that for very short periods after a visual presentation these limitations were also not imposed, and he distinguished a sensory register or echoic store as providing yet a third form of memory.

Subsequently, researchers focusing on long-term memory distinguished different types of long-term memories with differing characteristics. In 1953 William Scoville (1957) removed the hippocampus and surrounding areas of the medial temporal lobe in a patient known as H.M. in a successful attempt to ameliorate his severe epilepsy. The surgery, however, left H.M. with severe amnesia both for information acquired postsurgery (anterograde amnesia) and for periods prior to the surgery (graded retrograde amnesia). H.M. subsequently became a subject in numerous memory studies, and one surprising result was that he can learn new skills, although he never remembers that he had acquired the skill (Corkin 1968). This provided the first evidence of a dissociation between what has come to be called declarative or explicit memory and procedural or implicit memory (Cohen and Squire 1980).

Endel Tulving (1972), relying exclusively on behavioral evidence, introduced a distinction within explicit memory between "two parallel and partially overlapping information-processing systems," which he termed episodic and semantic memory. Roughly speaking, he construed episodic memory as memory of particular experiences and semantic memory as memory of facts. In his initial formulation, Tulving emphasized different tasks that tapped episodic and semantic memories. One experiment tested episodic memory by measuring recognition of words presented in a listlearning task. Either 1 hour or 7 days later, semantic memory was tested by asking the same subjects to fill in the missing letters in incomplete words, such as _o_ma_c. Although completing such a word fragment can 
be difficult, it was rendered much easier if the target word (here, dogmatic) was on the list used earlier in the episodic-memory task. Success in fragment completion remained roughly constant over the 1-week interval, whereas recognition performance declined dramatically. Such dissociation between results was construed as evidence that the psychological processes were independent. In a further analysis, Tulving established that the probability that subjects could complete a given fragment was stochastically independent of the probability that they could recognize it as having been on the study list (Tulving, Schacter, and Stark 1982), providing additional evidence that different operations were involved in the different types of tasks.

Over time Tulving came to emphasize more the type of knowledge included in episodic and semantic memories. Episodic memory involves retrieving episodes in a person's own life, whereas semantic memory is concerned with information that typically is retrieved independently of recalling the time and place in which it was acquired (e.g., word meanings, general knowledge, scientific facts). Although the distinction between episodic and semantic memory was initially developed relying on behavioral evidence, Tulving found powerful confirming evidence from neuropsychological patients. H.M.'s deficits involved both episodic and semantic memory, but another patient, K.C., who suffered a severe closed-head injury in 1981, lost all episodic memory, retrograde and anterograde, while retaining much of his semantic memory, including facts about his own life. Moreover, with extensive training, K.C. has been able to learn both new skills and new semantic information. Episodic memory is thus dissociated from semantic memory. (Vargha-Khadem et al. [1997] describe individuals who have been successful in learning information in school despite lacking episodic memory.)

In addition to differentiating types of memory phenomena, researchers have often attributed them to different components in a processing pathway. Thus, shortly after the differentiation of the sensory register, shortterm memory, and long-term memory, Richard Atkinson and Richard Shiffrin (1968) introduced a mathematical model in which control processes directed the transfer of information from the sensory register to short-term and then to long-term memory. Similarly, Tulving (1985) proposed a monohierarchical arrangement of long-term memory systems in which semantic memory relies on the processing in the procedural system and the episodic system relies on the processing of the semantic system.

For Tulving, the stochastic independence of performance on semantic and episodic tasks indicated that the episodic system was nearly, if not completely, distinct from the semantic system (from which it received inputs). While this has become the majority view, other researchers have dissented and proposed the components of processing approach (Roediger, 
Buckner, and McDermott 1999). On this account, different memory tasks do not tap largely independent systems but different operations from a common pool of cognitive operations. They appeal to neuroimaging studies showing that word-stem completion after priming produces activation in the same areas (visual cortex, left frontal opercular cortex, anterior cingulate, supplemental motor areas, and premotor and motor cortex) as word-stem completion without priming, but with reduced activity in visual cortex. This result seems problematic from a memory systems' view, according to which word-stem completion taps the semantic system, while priming taps the implicit system. Finally, if subjects are asked to complete the stem with a word they explicitly remember as one from the previously studied list, a task that should tap the episodic system, all the areas active on the word-stem completion remain active, and two additional areas, medial parietal cortex and right anterior prefrontal cortex, are engaged. Henry Roediger et al. (1999) thus reject the proposal of independent systems and argue that the mind selectively draws upon different component operations for different tasks. (For more detailed analysis of this debate, see Bechtel 2008.)

4. Looking High and Low for Operations. Neither Roediger et al. (1999) nor other advocates of processing accounts specify what operations figure in different memory tasks. One of the major methodologies of cognitive science is computational modeling, and modeling does require specifying operations through which the behavior is performed. Essentially, two ideas about operations have dominated computational modeling in cognitive science - syntactic processing of symbols and spreading activation between neuronlike units.

Symbol-processing models were developed by analogy with activities in which human agents manipulate symbols. Alan Turing's (1936) idea of a computational device using a finite state component to read and write symbols on a tape was inspired by the processes by which human beings, known as computers, performed complex computations by applying a set of learned procedures to numbers written on the page and writing new numbers on the page. The Turing machine's finite-state device that reads the current symbol on the tape and applies one of a finite set of rules to either replace the symbol or move the head and change its internal state models a human applying a finite set of rules to previously written symbols, with the tape representing the potentially infinite paper on which the human can write. The models of symbolic computational modeling essentially took the interaction between an agent and external memory as a model for operations in the head of the agent. In constructing the first functioning computational model, Logic Theorist (designed to prove theorems from Principia Mathematica), Allen Newell and Herbert Simon 
(1956) went so far as to have Simon and his children carry out the operations specified in the program. During the period other researchers in the nascent field of artificial intelligence pursued a different approach, one using networks of simple processing units that summed input and generated an output. After a period of relative neglect, this approach reemerged with the new-connectionists in the 1980s (Bechtel and Abrahamsen 2002).

Although it is appropriate to develop mechanistic models by appealing to analogies with operations performed in other mechanisms, there are reasons for suspecting that symbolic models are looking too high and connectionist models too low to identify the operations invoked in cognitive systems. The situation might be compared to the state of fermentation research in the late nineteenth century. By describing the potential intermediates formed in the process of fermentation as themselves undergoing fermentations, physiologists looked too high. They provided little explanatory gain, since researchers were appealing to the phenomenon to be explained to describe the operations that were to provide the explanation. In contrast, by focusing on the elemental composition of sugar and alcohol and appealing to operations of adding or deleting atoms to explain organic processes such as fermentation, chemists focused too low. The chemists clearly appealed to operations on components in a mechanism to explain the phenomenon, but this approach was underconstrained. Researchers lacked principles for determining which operatons were really possible. The major conceptual advance that led directly to the development of biochemistry as an autonomous discipline between the level of physical chemistry and physiology was the discovery that organic compounds are built of groups of atoms (e.g., amino groups $\left[\mathrm{NH}_{3}{ }^{+}\right]$, hydroxyl groups $\left[\mathrm{OH}^{-}\right]$, phosphate groups $\left.\left[\mathrm{PO}_{4}^{--}\right]\right)$. The basic operations of biochemistry were then recognized as involving the addition, deletion, or moving of such groups (phosphorylation and dephosphorylation involve adding or deleting phosphate groups from a substrate), operations frequently catalyzed by an appropriate enzyme (e.g., kinases in the case of phosphorylation). These operations were characterized in a vocabulary different from those used for the phenomena itself or the atoms that comprised the groups.

5. Seeking Guidance from the Brain: Heuristic Identity Theory. The challenge for cognitive psychology, including computational modeling, is to identify the appropriate operations for explaining mental phenomena. Theorists who have tried to develop comprehensive models of cognition such as SOAR (State, Operator, and Result; Newell 1990) and ACT (Adaptive Control of Thought; Anderson 1990) have faced this problem and have typically tried to build up from primitive symbol manipulations 
to more complex ones in order to generate appropriate cognitive behavior. Increasingly, such theorists have appealed to what is known about the brain to constrain their models (Newell, in particular, drew on temporal constraints). But until the development of neuroimaging techniques, such as positron emission tomography (PET) and functional magnetic resonance imaging (fMRI), there was limited information available from the brain to assist psychologists interested in identifying the operations underlying cognition. (There have also been principled arguments, based on the alleged multiple realizability of mental processes, against use of neural evidence to guide psychology. See Bechtel and Mundale 1999 and Shapiro 2004 for arguments defusing the import of multiple realizability claims.)

Often neuroimaging is construed as simply revealing where mental operations occur in the brain. While such knowledge can be useful, for the issues addressed in this article the real payoff is in providing guidance to characterizing the operations themselves. This requires identifying the mental operations with brain processes, but unlike traditional identity theory, the focus is on using the differences between what is known about the processes under each description as a discovery heuristic to revise the other. (Accordingly, McCauley and Bechtel [2001], speak of heuristic identity theory.) One way in which identity claims can be informative is when operations involved in two apparently different cognitive phenomena are localized in the same brain area. This prompts investigation of what operations the two phenomena could share in common. Another is when multiple brain areas are shown to be involved in a cognitive phenomenon and the neural pathways between these areas can be elicited. This prompts the attempt to specify operations for each area.

To make this proposal concrete, I consider three examples in which functional neuroimaging is providing new insights into the operations involved in memory. In these cases, the insight comes from first identifying a memory system with one brain area and then discovering that that area is involved in memory tasks associated with a competing memory system or brain areas involved in activities not usually associated with memory. This provides additional constraint to guide the search for the mechanisms involved in memory.

The first example follows up on a pioneering neuroimaging study memory in which Tulving et al. (1994) linked the encoding of episodic memories (which requires retrieval of semantic information) with left prefrontal cortex and retrieval of episodic memories with right prefrontal cortex. A first challenge to this claim arose when James Brewer et al. (1998) substituted pictures for the verbal stimuli used by Tulving et al. and elicited right prefrontal activation on episodic encoding tasks. A second challenge followed when Larry Squire and Stuart Zola (1998) discovered that lesions in left prefrontal cortex resulted in deficits in episodic retrieval tasks that 
required remembering how one acquired the memory. Although these studies were inconsistent with Tulving et al.'s claim, Emrah Düzel et al. (1999) reported new imaging studies that supported the original left/right distinction between semantic and episodic retrieval. M. Natasha Rajah and Anthony McIntosh (2005), however, reanalyzed their data using structural equation modeling (SEM), a form of multivariate analysis that interprets correlations in activation between different areas as evidence that those areas are linked in a common causal network. Even under the most favorable conditions, their analysis failed to support the claim that distinct networks were involved in semantic and episodic retrieval tasks.

A second example challenges the idea that short-term memory supplies the input to long-term memory, as embraced in the Atkinson and Shiffrin model (1968). Timothy Shallice and Elizabeth Warrington (1970) had reported on patients with impaired short-term but intact long-term memory for the same materials on which their short-term memory was impaired. This supported different loci for short- and long-term memory systems, with medial-temporal lobe (MTL) structures (hippocampus, perirhinal, parahippocampal, and entorhinal cortices) viewed as essential for the encoding of long-term episodic memories and perisylvian regions as figuring in phonological short-term memory. Charan Ranganath and Robert Blumenfeld (2005), however, report that with both monkeys and humans, when complex and novel stimuli are employed, MTL structures figure in short-term memory. They also report that long-term memory tasks with a required phonological component also engage the perisylvian region. They thereby claim that the full range of findings undermines the distinction between systems.

A third example questions the very distinction between memory and other mental phenomena. When Tulving et al. (1994) identified areas in left prefrontal cortex as involved in semantic retrieval and episodic encoding, they noted that the areas were very close to those identified in earlier PET studies as performing semantic processing in a linguistic task (i.e., generating a verb to use with a noun). They argued that even the verb-generate task should be viewed as an episodic encoding task. In contrast, John Gabrieli, Russell Poldrack, and John Desmond (1998), reviewing a variety of studies involving the region in left prefrontal cortex, concluded that the "operations may be the same whether they are considered in the context of language, working memory, episodic memory, or implicit memory. The left prefrontal cortex thus serves as a crossroads between meaning in language and memory" (912). This suggests that memory processes are more integrated with other cognitive activities than previously assumed. Support for this comes from the growing recognition, based on a variety of studies demonstrating the generation of false memories, that recollection is a constructive process, not an elicitation of a 
stored engram. Accordingly, Randy Buckner and Daniel Schacter (2004) have proposed focusing on how an overall cognitive system could reconstruct the past. They note in particular evidence that the more demanding the task, the more anterior are the frontal areas that are activated. In contrast, regions in left parietal cortex seem to track "the perception or decision that information is old," whether or not the information provides a correct response to the current memory task. They construe the frontal areas as directing attention toward more posterior brain regions involved in initial information processing.

On a view such as Buckner and Schacter are proposing, memory is conceived not as an autonomous mental capacity but as actively reengaging cognitive operations used in other cognitive tasks. This seems, on the one hand, to deny the existence of memory as a distinct phenomenon (or set of phenomena) with its own mechanism (or mechanisms). On the other hand, though, it potentially enables memory research to draw upon advances that have been made in decomposing the operations involved in visual and other sensory processing using single-cell recording in nonhuman animals. The characterization of the information processing occurring in these brain areas may require revision when it is recognized that they subserve not just processing sensory stimuli but also memory and other uses of that information. Nonetheless, the attempt to identify memory processes with brain areas will have served a vital heuristic role in reconceptualizing the phenomena being explained and identifying new resources for characterizing the operations involved (see Bechtel 2008 for further discussion).

6. Conclusion. Psychologists, like biologists, frequently appeal to mechanisms in explaining phenomena. Unlike biologists, who often can identify operations in mechanisms by tracing material transformations, psychologists investigating information processing have faced a greater challenge in identifying operations. The ability to identify brain areas with cognitive operations, though, provides a heuristic that is proving of great use. In the case of memory, it is revealing that different memory phenomena that were attributed to different systems employ the same brain areas and that memory tasks rely on brain areas that also figure in other cognitive tasks. This does not directly solve the problem of identifying operations but focuses attention on what might be common operations invoked in these different psychological phenomena. 
The Psychology of Learning and Motivation: Advances in Research and Theory. New York: Academic, 89-195.

Bechtel, William (2006), Discovering Cell Mechanisms: The Creation of Modern Cell Biology. Cambridge: Cambridge University Press.

- (2008), Mental Mechanisms: Philosophical Perspectives on Cognitive Neuroscience. London: Routledge.

Bechtel, William, and Adele Abrahamsen (2002), Connectionism and the Mind: Parallel Processing, Dynamics, and Evolution in Networks. 2nd ed. Oxford: Blackwell.

$\rightarrow$ Bechtel, William, and Jennifer Mundale (1999), "Multiple Realizability Revisited: Linking Cognitive and Neural States", Philosophy of Science 66: 175-207.

Bechtel, William, and Robert C. Richardson (1993), Discovering Complexity: Decomposition and Localization as Strategies in Scientific Research. Princeton, NJ: Princeton University Press.

$\rightarrow$ Brewer, James B., Zuo Zhao, John E. Desmond, Gary H. Glover, and John D. E. Gabrieli (1998), "Making Memories: Brain Activity That Predicts How Well Visual Experience Will Be Remembered", Science 281: 1185-1187.

Broadbent, Donald (1958), Perception and Communication. London: Pergamon.

$\rightarrow$ Bruner, Jerome S., and Cecile C. Goodman (1947), "Value and Need as Organizing Factors in Perception", Journal of Abnormal and Social Psychology 42: 33-34.

Buckner, Randy L., and Daniel L. Schacter (2004), "Neural Correlates of Memory's Successes and Sins", in Michael S. Gazzaniga (ed.), The Cognitive Neurosciences III. Cambridge, MA: MIT Press, 739-752.

$\rightarrow$ Chomsky, Noam (1956), "Three Models for the Description of Language", Transactions on Information Theory 2: 113-124.

$\rightarrow-(1959)$, "Review of Verbal Behavior", Language 35: 26-58.

$\rightarrow$ Cohen, Neal J., and Larry R. Squire (1980), "Preserved Learning and Retention of PatternAnalyzing Skill in Amnesia: Dissociation of Knowing How and Knowing That", Science 210: $207-210$.

$\rightarrow$ Corkin, Suzanne (1968), “Acquisition of Motor Skill after Bilateral Medial Temporal-Lobe Excision", Neuropsychologia 6: 255-265.

Craver, Carl (2007), Explaining the Brain: What a Science of the Mind-Brain Could Be. New York: Oxford University Press.

Cummins, Robert (2000), “'How Does It Work?' versus 'What Are the Laws?': Two Conceptions of Psychological Explanation", in Frank Keil and Robert Wilson (eds.), Explanation and Cognition. Cambridge, MA: MIT Press, 117-144.

Darden, Lindley (2005), "Relations among Fields: Mendelian, Cytological and Molecular Mechanisms", Studies in the History and Philosophy of Biological and Biomedical Science 36: $357-371$.

$\rightarrow$ Düzel, Emrah, Roberto Cabeza, Terence W. Picton, Andrew P. Yonelinas, Henning Scheich, Hans-Jochen Heinze, and Endel Tulving (1999), "Task-Related and Item-Related Brain Processes of Memory Retrieval", Proceedings of the National Academy of Sciences (USA) 96: 1794-1799.

Ebbinghaus, Hermann (1885), Über das Gedächtnis: Untersuchungen zur experimentellen Psychologie. Leipzig: Duncker \& Humblot.

$\rightarrow$ Gabrieli, John D. E., Russell A. Poldrack, and John E. Desmond (1998), "The Role of Left Prefrontal Cortex in Language and Memory", Proceedings of the National Academy of Sciences, USA 95: 906-913.

Hebb, Donald O. (1949), The Organization of Behavior. New York: Wiley.

James, William (1890), Principles of Psychology. New York: Holt.

$\rightarrow$ Machamer, Peter, Lindley Darden, and Carl Craver (2000), "Thinking about Mechanisms", Philosophy of Science 67: 1-25.

$\rightarrow$ McCauley, Robert N., and William Bechtel (2001), "Explanatory Pluralism and Heuristic Identity Theory", Theory and Psychology 11: 736-760.

$\rightarrow$ Miller, George A. (1956), "The Magical Number Seven, Plus or Minus Two: Some Limits on Our Capacity for Processing Information”, Psychological Review 63: 81-97.

Miller, George A., Eugene Galanter, and Karl Pribram (1960), Plans and the Structure of Behavior. New York: Holt. 
Neisser, Ulric (1967), Cognitive Psychology. New York: Appleton-Century-Crofts.

Newell, Allen (1990), Unified Theories of Cognition. Cambridge, MA: Harvard University Press.

$\rightarrow$ Newell, Allen, and Herbert A. Simon (1956), "The Logic Theory Machine", IRE Transactions on Information Theory 3: 61-79.

$\rightarrow$ Peterson, L. R., and M. J. Peterson (1959), "Short-Term Retention of Individual Verbal Items", Journal of Experimental Psychology 58: 193-198.

$\rightarrow$ Rajah, M. Natasha, and Anthony R. McIntosh (2005), "Overlap in the Functional Neural Systems Involved in Semantic and Episodic Memory Retrieval", Journal of Cognitive Neuroscience 17: 470-482.

$\rightarrow$ Ranganath, Charan, and Robert S. Blumenfeld (2005), "Doubt about Double Dissociations between Short- and Long-term Memory", Trends in Cognitive Science 9: 374-380.

Roediger, Henry L., Randy L. Buckner, and Kathleen B. McDermott (1999), "Components of Processing", in Jonathan K. Foster and Marko Jelicic (eds.), Memory: Systems, Process, or Function, Oxford: Oxford University Press, 32-65.

$\rightarrow$ Scoville, W. B., and B. Milner (1957), "Loss of Recent Memory after Bilateral Hippocampal Lesions", Journal of Neurology, Neurosurgery, and Psychiatry 20: 11-21.

$\rightarrow$ Shallice, Timothy, and Elizabeth K. Warrington (1970), "Independent Functioning of Verbal Memory Stores: A Neuropsychological Study", Quarterly Journal of Experimental Psychology 22: 261-273.

Shannon, Claude E. (1948), “A Mathematical Theory of Communication”, Bell System Technical Journal 27: 379-423, 623-656.

Shapiro, Lawrence (2004), The Mind Incarnate. Cambridge, MA: MIT Press.

Sperling, George (1960), "The Information Available in Brief Visual Presentations", Psychological Monographs 74: 1-29.

$\rightarrow$ Squire, Larry R., and Stuart M. Zola (1998), "Episodic Memory, Semantic Memory, and Amnesia", Hippocampus 8: 205-211.

$\rightarrow$ Sternberg, Saul (1966), "High-Speed Scanning in Human Memory”, Science 153: 652-654.

$\rightarrow$ Teigen, K. H. (2002), "One Hundred Years of Laws in Psychology", American Journal of Psychology 115: 103-118.

Tulving, Endel (1972), "Episodic and Semantic Memory", in Endel Tulving and Wayne Donaldson (eds.), Organization of Memory. New York: Academic Press, 381-403.

$\rightarrow-(1985)$, "How Many Memory Systems Are There?" American Psychologist 40: 385398.

$\rightarrow$ Tulving, Endel, Shitu Kapur, Fergus I. M. Craik, Morris Moscovitch, and Sylvain Houle (1994), "Hemispheric Encoding/Retrieval Asymmetry in Episodic Memory: Positron Emission Tomography Findings", Proceedings of the National Academy of Sciences (USA) 91: 2016-2020.

$\rightarrow$ Tulving, Endel, Daniel L. Schacter, and Heather A. Stark (1982), "Priming Effects in WordFragment Completion Are Independent of Recognition Memory", Journal of Experimental Psychology: Human Learning and Memory 8: 336-342.

$\rightarrow$ Turing, Alan (1936), "On Computable Numbers, with an Application to the Entscheidungsproblem", Proceedings of the London Mathematical Society, 2nd ser., 42: 230265.

$\rightarrow$ Vargha-Khadem, Faraneh, David G. Gadian, Kate E. Watkins, Alan Connelly, Wim Van Paesschen, and Mortimer Mishkin (1997), "Differential Effects of Early Hippocampal Pathology on Episodic and Semantic Memory", Science 277: 376-380.

$\rightarrow$ Waugh, Nancy C., and Donald A. Norman (1965), "Primary Memory", Psychological Review 72: 89-104. 